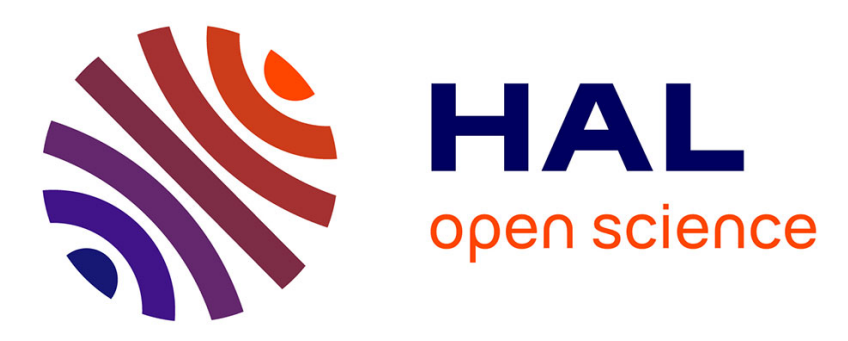

\title{
Giant field enhancement in electromagnetic Helmholtz nanoantenna
}

Paul Chevalier, Patrick Bouchon, Jean-Jacques Greffet, Jean-Luc Pelouard, Riad Hadar, Fabrice Pardo

\section{- To cite this version:}

Paul Chevalier, Patrick Bouchon, Jean-Jacques Greffet, Jean-Luc Pelouard, Riad Hadar, et al.. Giant field enhancement in electromagnetic Helmholtz nanoantenna. Physical Review B: Condensed Matter and Materials Physics (1998-2015), 2014, 90, pp.195412. 10.1103/PhysRevB.90.195412 . hal01082836

\section{HAL Id: hal-01082836 https://hal.science/hal-01082836}

Submitted on 14 Nov 2014

HAL is a multi-disciplinary open access archive for the deposit and dissemination of scientific research documents, whether they are published or not. The documents may come from teaching and research institutions in France or abroad, or from public or private research centers.
L'archive ouverte pluridisciplinaire HAL, est destinée au dépôt et à la diffusion de documents scientifiques de niveau recherche, publiés ou non, émanant des établissements d'enseignement et de recherche français ou étrangers, des laboratoires publics ou privés. 


\title{
Giant field enhancement in electromagnetic Helmholtz nanoantenna
}

\author{
Paul Chevalier, ${ }^{1,2}$ Patrick Bouchon, ${ }^{2, *}$ Jean-Jacques Greffet, ${ }^{3}$ \\ Jean-Luc Pelouard, ${ }^{1}$ Riad Haïdar, ${ }^{2,4}$ and Fabrice Pardo ${ }^{1}$ \\ ${ }^{1}$ Laboratoire de Photonique et de Nanostructures (LPN-CNRS), Route de Nozay, 91460 Marcoussis, France \\ ${ }^{2}$ ONERA, The French Aerospace Lab, 91761 Palaiseau, France \\ ${ }^{3}$ Laboratoire Charles Fabry, Institut d'Optique, 91127 Palaiseau, France \\ ${ }^{4}$ École Polytechnique, Département de Physique, 91128 Palaiseau, France
}

(Dated: October 20, 2014)

\begin{abstract}
Inspired by the acoustic Helmholtz resonator, we propose a slit-box electromagnetic nanoantenna able to concentrate the energy of an incident beam into surfaces a thousand times smaller than with a classical lens. This design produces a giant electric field enhancement throughout the slit. The intensity enhancement reaches $10^{4}$ in the visible up to $10^{8}$ in the $\mathrm{THz}$ range even with focused beams thanks to an omnidirectional reception. These properties could target applications requiring extreme light concentration, such as SEIRA, non-linear optics and biophotonics.
\end{abstract}

\section{INTRODUCTION}

Optical antennas are very useful to funnel light from free space into deep-subwavelength volumes resulting in large electric field enhancements ${ }^{1-8}$. They have a wide range of applications including surface enhanced Raman scattering (SERS $)^{9}$, photovoltaics and photodetection ${ }^{10}$, non-linear optics ${ }^{11}$, bio-sensing ${ }^{12}$, surface enhancement infrared absorption (SEIRA) ${ }^{13-15}$, and thermal emis$\operatorname{sion}^{16}$. The important properties of an antenna are its cross-section that determines its coupling to the external field, its angular acceptance that allows to further enhance the field with a focused beam, and the volume in which the field is enhanced, which is critical for light matter interactions based applications. Intensity enhancement up to $10^{3}$ was observed using subwavelength particles $^{17}$ and an enhancement of $10^{8}$ has been demonstrated in atomic thin gaps in metal for millimeter waves ${ }^{18}$. Inspired by radiofrequency designs, various nanoantennas have been engineered to improve the field enhancement in larger volumes. For instance, Yagi-Uda antenna have been adapted to optics, but are limited by their directionality ${ }^{19-21}$. Bow-tie antenna are less directional, but only enhance the field in hot $\operatorname{spots}^{22,23}$. Eventually, quarter-wave antennas such as slits or metal-insulatormetal patches have been shown to efficiently collect light thanks to a funneling effect with a near- $\lambda$ cross section and a large angular acceptance ${ }^{24-27}$. Nonetheless, the enhancement of the field is mainly limited by the aperture size of these quarter-wave antenna, and is not homogeneous within their length. Recently, inspired by acoustic design ${ }^{28}$, an optical Helmholtz resonator made of a metallic slit-box has been demonstrated ${ }^{29}$. The resonance wavelength $\lambda$ of the optical Helmholtz resonator is tuned independently by the slit (width: $w$, height: $h$ ) and the cavity.

In this paper, we show that it is able to omnidirectionally funnel all the light through its aperture regardless of its width, leading to a million-fold electric field intensity enhancement in a hot volume. Based on a quasistatic model, we demonstrate on a wide spectral

range $(1-100 \mu \mathrm{m})$ that the giant enhancement factor is obtained within the volume of the slit driven by the ratio $\lambda^{2} /(h w)$. This enhancement factor only depends slightly on the angle of incidence which permits to use this antenna at the focal spot of a classical lens to further strongly enhance the electric field intensity. This giant field intensity enhancement should be instrumental for devices based on SEIRA or nonlinear optics which use focused beam.

\section{OPTICAL HELMHOLTZ RESONATOR}

(a)

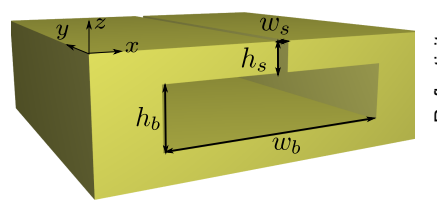

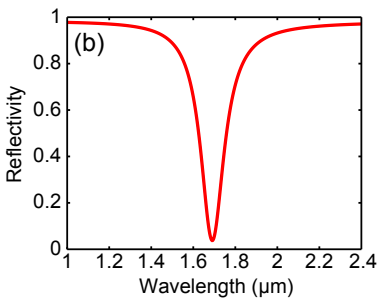

FIG. 1. (a) Two-dimensional Helmholtz resonator infinite in the $\mathbf{y}$ direction, with a slit of height $h_{s}$ and width $w_{s}$ and a box of width $w_{b}$ and height $h_{b}$. (b) Reflectivity spectrum of a grating of Helmholtz slit-box resonator with a period $d=$ $0.8 \mu \mathrm{m}$ for a normally incident plane wave. The Helmholtz resonator has the following parameters: $w_{\mathrm{b}}=49 \mathrm{~nm}, h_{\mathrm{b}}=$ $49 \mathrm{~nm}, w_{\mathrm{s}}=2.5 \mathrm{~nm}$ and $h_{\mathrm{s}}=12 \mathrm{~nm}$ and exhibits an almost total absorption for $\lambda_{r}=1.7 \mu \mathrm{m}$.

The optical Helmholtz resonator is presented in Fig. 1(a), it consists of a box (height $h_{b}$, width $w_{b}$ ) and a slit (height $h_{s}$, width $w_{s}$ ) and is long enough along the $\mathbf{y}$ direction to be considered as infinite. The structure is made of gold whose dielectric function is computed from a Drude model $\varepsilon_{\mathrm{Au}}(\lambda)=1-\left[\left(\lambda_{p} / \lambda+i \gamma\right) \lambda_{p} / \lambda\right]^{-1}$ which fits well the experimental data in the infrared domain for $\lambda_{p}=159 \mathrm{~nm}$ and $\gamma=0.0077^{30}$.

Exact Maxwell computations ${ }^{31}$ of this slit-box resonator are performed for a first structure such that $w_{b}=49 \mathrm{~nm}, h_{b}=49 \mathrm{~nm}, w_{s}=2.5 \mathrm{~nm}$ and $h_{s}=12 \mathrm{~nm}$ 

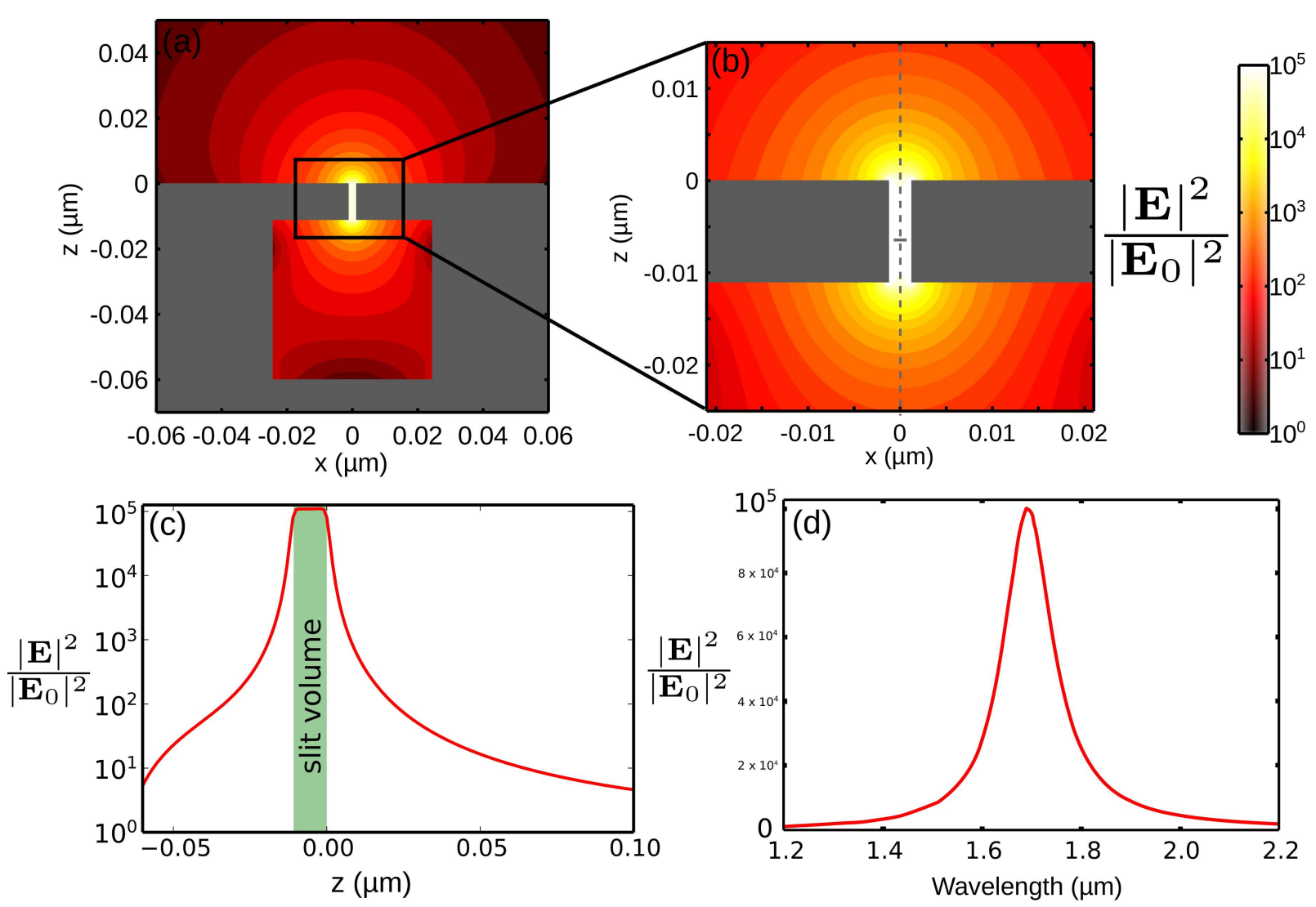

FIG. 2. The electric field intensity enhancement is shown at different scales for an incident normally incident plane wave. (a) Field intensity enhancement in the Helmholtz resonator at $\lambda=1.7 \mu \mathrm{m}$. (b) Close view in the vicinity of the slit. (c) Field intensity enhancement along the $z$-axis, at the center of the structure $(x=0)$. A rather homogeneous enhancement of $10^{5}$ is found in the whole slit volume. (d) The field intensity enhancement in the center of the slit is plotted at different wavelengths. We see that $\lambda=1.7 \mathrm{\mu m}$ is a resonance wavelength that provide a maximal enhancement. The quality factor of this resonance is about 10 .

periodically repeated with a period $d=800 \mathrm{~nm}$. Its reflectivity spectrum is plotted on Fig. 1(b) between $1 \mu \mathrm{m}$ and $2.4 \mu \mathrm{m}$. It exhibits a nearly total absorption resonance at $\lambda_{r}=1.7 \mu \mathrm{m}$.

\section{FIELD ENHANCEMENT FROM NEAR IR TO THZ}

\section{A. Examples of giant field enhancement}

The distribution of the electric intensity $\left(|\mathbf{E}|^{2}\right)$ normalized by the incident intensity $\left(\left|\mathbf{E}_{0}\right|^{2}\right)$ in the structure is presented in Figs. 2(a)-2(b) at different zoom levels and shows that a field intensity enhancement of $10^{5}$ is reached with this structure. In Fig. 2(c), it is shown that this enhancement is confined to the slit volume: the field intensity enhancement stays uniform along the height of the slit, but outside its volume the electric field intensity quickly drops by losing 4 orders of magnitude over about $50 \mathrm{~nm}$. Noteworthily, this profile of the electric field departs from the behaviour of a Fabry-Perot slit, and it adds to the fact that the slit-box resonator does not exhibit harmonics features ${ }^{32}$. The quality factor is $Q \simeq 10$ for a resonance wavelength of $\lambda_{R}=1.7 \mu \mathrm{m}$ as shown in Fig. 2(d).

Very strong field intensity enhancements can be reached not only in the infrared range, but in the whole spectrum from visible to $\mathrm{THz}$ domain. To illustrate this, two other structures have been designed to exhibit a strong field enhancement at the wavelengths of $15 \mu \mathrm{m}$ and $200 \mu \mathrm{m}$. The second structure is made of a square box where $w_{\mathrm{b}}=h_{\mathrm{b}}=485 \mathrm{~nm}$ and $w_{\mathrm{s}}=2 \mathrm{~nm}$, $h_{\mathrm{s}}=30 \mathrm{~nm}$ is simulated on a periodic grating (period 
$d=3 \mu \mathrm{m})$ and exhibits a total absorption at a resonance wavelength of $15 \mu \mathrm{m}$ where a $10^{6}$ intensity enhancement is observed. Another structure with a box such that $w_{\mathrm{b}}=h_{\mathrm{b}}=5.7 \mu \mathrm{m}, w_{\mathrm{s}}=4 \mathrm{~nm}, h_{\mathrm{s}}=80 \mathrm{~nm}$, and $d=50 \mu \mathrm{m}$ shows $10^{8}$-fold intensity enhancement at a resonance wavelength of $200 \mu \mathrm{m}$.

Theses enhancement factors for the Helmholtz structures can be compared to the literature showing theoretical predictions of high field enhancement in large volumes as we do not compare to enhancements occurring in hot spots. In the near infrared, an enhancement factor of $10^{5}$ is observed whereas the literature shows usually a $10^{3}$ enhancement factor for the near IR or visible wavelengths $8,13,15,17$. In the far IR we demonstrate a $10^{6}$ enhancement factor compared to $10^{4}$ in the literature. ${ }^{33}$, and finally we observe $10^{8}$ vs. $10^{6}$ in the $\mathrm{THz}$ domain ${ }^{34}$.

As a matter of fact, these record values are obtained thanks to exact electromagnetic computations, using a realistic permittivity of gold ${ }^{30}$. However, the geometry of the structure exhibits high aspect ratios with nanometric slits. A real structure might thus yield lower enhancement values due to technological imperfections.

\section{B. Model for the field intensity enhancement}

It has been shown that the slit-box structure is fairly well described by a quasistatic model: the slit behaves as a capacitor and the box as an inductance ${ }^{29}$. Thus, for an arbitrary length $T$ of the structure in the $\mathbf{y}$ direction, the capacitance of the slit can be written as $C_{\text {slit }}=\varepsilon_{0} \varepsilon_{s} \frac{h_{s} T}{w_{s}}$ and the inductance of the cavity as $L_{\text {box }}=\mu_{0} \frac{w_{b} h_{b}}{T}$. The resonance wavelength is eventually expressed as $\lambda_{r} \simeq 2 \pi \sqrt{\varepsilon_{s} w_{b} h_{b}\left(h_{s}+w_{s}\right) / w_{s}}$ where $\varepsilon_{s}$ is the permittivity of the dielectric material filling the slit. However, when using Helmholtz structures made of real metal at near-infrared frequencies, the previous model should be corrected as:

$$
\lambda_{r}=2 \pi A \sqrt{\varepsilon_{s} w_{b} h_{b} \frac{h_{s}+K w_{s}}{w_{s}}}+B \lambda_{p} .
$$

The coefficients have been fitted on the spectral range between $1.5 \mu \mathrm{m}$ and $15 \mu \mathrm{m}$ as $A=1.16, B=4.4$ and $K=1.1$.

We now derive the enhancement factor of the resonator. Let us first introduce the quality factor of the resonator which links the energy dissipated during one period to the energy stored in the resonator:

$$
\frac{Q_{n r}}{2 \pi}=\frac{U_{\text {stored }}}{U_{\text {dissipated per period }}}
$$

We now give an explicit form of the energy stored in the resonator by evaluating the maximum energy stored in the capacitor. Given the link between the electric field $|\mathbf{E}|$ amplitude and the maximum difference of electric potential $V$ in the capacitor: $|V| \simeq|\mathbf{E}| w_{s}$, the stored energy in the resonator for one spatial period along the $x$-direction and a length $T$ along the $y$-direction is:

$$
U_{\text {stored }}=\frac{1}{2} C_{\text {slit }}|\mathbf{E}|^{2} w_{s}^{2}=\frac{\varepsilon_{0} \varepsilon_{s}|\mathbf{E}|^{2} h_{s} w_{s} T}{2}
$$

We now express the energy dissipated during one cycle. In stationary regime, the power dissipated in a spatial period of area $d T$ is equal to the incident power given by:

$$
P_{\text {incident }}=\frac{\left|\mathbf{E}_{0}\right|^{2} d T}{2 Z_{0}},
$$

where $Z_{0}=\sqrt{\frac{\mu_{0}}{\varepsilon_{0}}}$ is the characteristic impedance of vacuum and $\mathbf{E}_{0}$ is the amplitude of the incoming plane wave.

In stationary regime, the incident power is equal to the dissipated power. It follows that the dissipated energy during one temporal period is given by:

$$
U_{\text {dissipated per period }}=\frac{\varepsilon_{0} A\left|\mathbf{E}_{0}\right|^{2} \lambda d T}{2}
$$

where $A$ is the absorption coefficient of the structure.

This allows to express the field intensity enhancement $G$ thanks to Eqs. $(1,2,4)$ :

$$
G=\frac{|\mathbf{E}|^{2}}{\left|\mathbf{E}_{0}\right|^{2}}=\frac{Q_{n r}}{2 \pi} \frac{A \lambda d}{\varepsilon_{s} h_{s} w_{s}}
$$

In the optimal situation where a perfectly impedancematched structure is designed $(A=1)$ and for which the cross section is maximum $(d=\lambda)$, the maximum field intensity enhancement $G_{\max }$ in the slit at the resonance can be expressed as:

$$
G_{\max }=\frac{\left|\mathbf{E}_{\max }\right|^{2}}{\left|\mathbf{E}_{0}\right|^{2}}=\frac{Q_{n r}}{2 \pi} \frac{\lambda^{2}}{\varepsilon_{s} h_{s} w_{s}}
$$

In figure 3 the enhancement factor has been plotted for variously shaped structures $\left(\varepsilon_{s}=1\right)$ exhibiting resonances from the near infrared to the far infrared and is plotted as a function of $G$. This figure shows the good agreement between this analytic model and the values obtained through numerical simulations in the different domains of frequencies.

It must be emphasized that at a given wavelength, the field enhancement is set by the area of the slit. Higher enhancement factor are obtained at larger wavelength and for smaller slits. The quality factor of the resonance for simulated structures was between 5 and 15 . The resonance wavelength being set by other geometric parameters $^{29}$, this model gives the rules to design a Helmholtz structure exhibiting a given field enhancement at a given wavelength within the simulated range. 


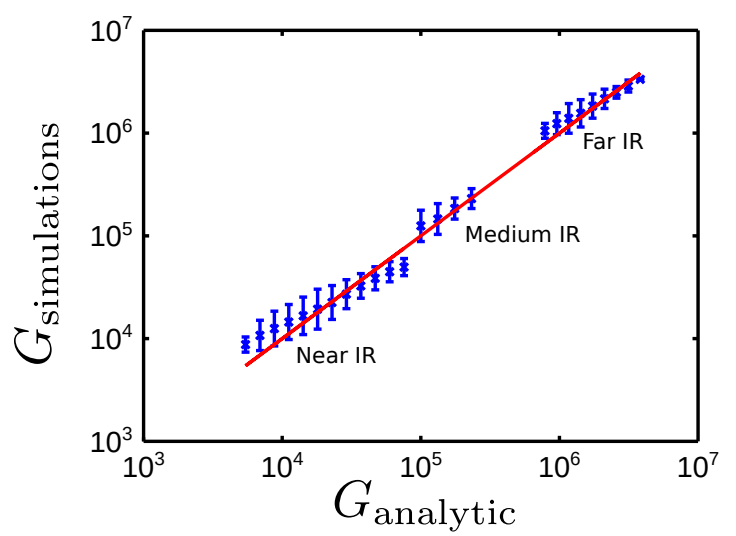

FIG. 3. Simulations for different size of structure have been realized with $\varepsilon_{s}=1$. For each structure the enhancement factor $G_{\text {simulations }}$ for a plane incident wave is computed and compared to the analytic model $G_{\text {analytic }}$ expressed in equation 5 as a function of the geometry. We see a strong correlation between the simulations results and the formula provided in the manuscript for the different domains of frequency. Three sets of simulations have been made, the first in the near IR domain ( $\lambda$ between $2 \mu \mathrm{m}$ and $8 \mu \mathrm{m}$ ), the second in the medium IR ( $\lambda$ between $10 \mu \mathrm{m}$ and $25 \mu \mathrm{m}$ ) and the last in the far IR domain ( $\lambda$ between $30 \mu \mathrm{m}$ and $80 \mu \mathrm{m})$.

\section{POLARIZATION INDEPENDENT DESIGN OF THE GIANT FIELD ENHANCEMENT}

A potential drawback of the $2 D$ resonator with a single slit introduced in ref. 29 is its polarization dependence. To make the Helmholtz structure independent to the light polarization on a normal incidence, one Helmholtz structure along the $x$-axis can be crossed with one structure along the $y$-axis as it can be seen in Fig. 4(a). For this structure with geometric dimensions similar as the structures presented above (in Figs. 1-2), the electromagnetic computations made with COMSOL multiphysics show that the resonance wavelength is red shifted from $1.7 \mathrm{\mu m}$ for the $2 D$ resonator to $1.8 \mu \mathrm{m}$ for the $3 D$ resonator.

At the resonance wavelength, the field intensity enhancement for a linearly polarized wave equally distributed between the $x$-axis and the $y$-axis is plotted in Figs. 4(b-c). In this situation the electric intensity is enhanced by at least 35000 (between two slit intersections) and up to $10^{5}$ at the intersection between the two slits.

When the light is normally incident and polarized along the $x$-axis, the field enhancement is of the same magnitude as in the $2 D$ structure and is localized in the slit along the $y$-direction. For a normally incident plane wave equally distributed along the two axis, one half of the energy correspond to the $x$-component of the electric field, and the other half correspond to its $y$-component. In this case, each component produces a slightly smaller intensity enhancement at the extremity of the slit compared to the half of the intensity enhancement provided by the $2 D$ structure, however the two contributions are summed at the intersection of the slits, showing that this

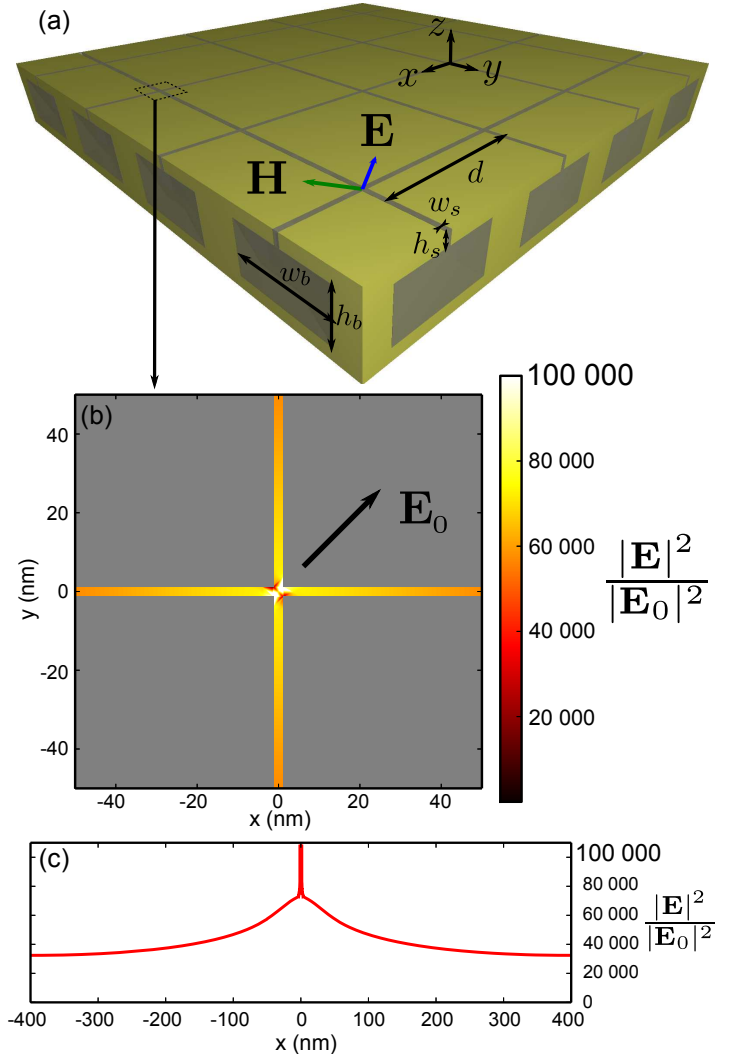

FIG. 4. (a) Polarization independent structure which is a combination of two crossed Helmholtz structures. For a polarization independent structure, having similar dimension as the structure studied above, the resonance wavelength is slightly shifted to $1.8 \mathrm{\mu m}$. (b) The field intensity enhancement is plotted at the middle of the slit height and near the intersection of the two structures, for the incident field $\mathbf{E}_{0}$ specified on the figure (equally distributed between the $x$ and $y$ axis). (c) The intensity enhancement along the $x$-axis and average along the $y$-axis is plotted for one period. It shows that the field enhancement is maximal at the intersection where a hotspot appears. Elsewhere inside the slit, the field intensity enhancement is above 35000 and increases up to 70000 .

design could still be used for strong intensity enhancement with fields with any polarization.

\section{ANGULAR ACCEPTANCE AND LIGHT FOCUSING}

The angular dependence of the enhancement is studied in Fig. 5. The general configuration of conical incidence is described in Fig. 5(a), the light is impinging on the antenna with an angle $\vartheta$ in the plane defined by the azimuthal angle $\varphi$ and is polarized so that the magnetic field is in the $y-z$ plane. The enhancement $\mathrm{G}$ as a function of both angles is plotted in Fig. 5(b). It remains above $5.10^{4}$ for angle of incidence below $35^{\circ}$ which demonstrates the broad acceptance of the resonator.

Due to the high angular tolerance of the structure, the 


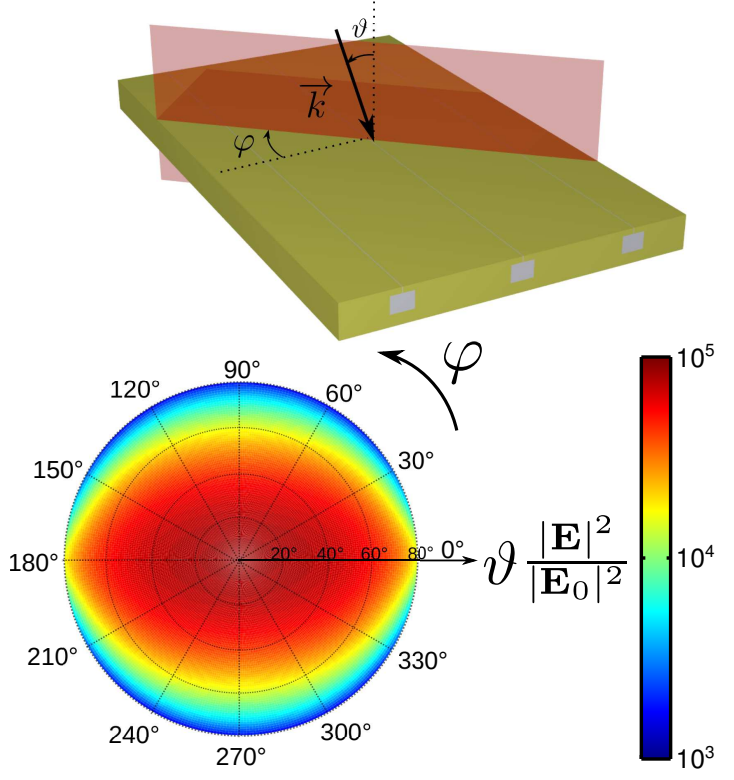

FIG. 5. The electric field intensity enhancement is computed for an incident plane wave (polarized parallel to the slits) with different angles of incidence and different planes of incidence. The structure is the same as described in the manuscript. (a) The plane of incidence is rotated by an angle $\varphi$, the angle of incidence is $\vartheta$. (b) The results of the field intensity enhancement is represented with polar coordinates on the right. The field intensity enhancement remains above $5 \times 10^{4}$ for angles of incidence below $40^{\circ}$, it is above $10^{4}$ for angle of incidence below $65^{\circ}$.

enhancement $G$ would only be slightly reduced when the structure is placed at the focal spot of a lens. In this case, the electric field intensity $|\mathbf{E}|^{2}$ would be similar to the one obtained if, beyond the limits of diffractive optics, a beam of given power was focused in a spot of area $\lambda^{2} / G$ instead of $\lambda^{2}$. Noteworthily, this intensity is uniformly obtained in a "hot volume" of height $h_{s}$ and area $\lambda w_{s} \gg \lambda^{2} / G$.

The behavior of the slit-box structure under a focused beam has been simulated ${ }^{32}$. The streamlines of the Poynting vector plotted at a large scale in Fig. 6(b) show that the incident energy is focused into a beam limited by diffraction (see Fig. 6(c)) where $90 \%$ of the energy is concentrated in a $\lambda$-wide spot (here the wavelength is $\lambda=1.7 \mu \mathrm{m})$. Next, in the near field $(z<500 \mathrm{~nm}$, see Fig. 6(d)), all the focused energy is funneled towards the opening of the resonators where it is absorbed on the metallic sidewalls. In fact, given the size of the focal spot, $60 \%$ of the total energy is absorbed by the central resonator while the remaining energy is equally absorbed by the two surrounding resonators. Since the incoming energy goes through the slit of the resonator, the electric field intensity is still strongly enhanced in the whole slit volume.

Focusing light onto the structure produces a stronger incident field that is further enhanced into the tiny slit by the mechanism described above ; the giant enhancement factor is also unchanged with focused beams. Thus, the resulting electric field intensity after focusing light onto one slit would be stronger than the one obtained for plane incident wave carrying the same energy.

\section{CONCLUSION}

The 2D optical Helmholtz resonator allows to funnel nearly all the incident energy through the arbitrarily narrow aperture of the slit leading to giant enhancement of the electric field in the whole slit volume, typically 2 orders of magnitude higher than previously demonstrated in the literature. It is well described by a quasistatic model where the slit behaves as a capacitor and the cavity as an inductance, and is only coupled to the free space through the capacitor. This model permits to derive the enhancement factor within the volume of the slit, as a function of the slit area and the quality factor. This enhancement factor only depends slightly on the angle of incidence which permits to use this antenna at the focal spot of a classical lens to further strongly enhance the electric field intensity. These appealing properties are very promising for light matter interactions such as in photodetection, SERS, SEIRA and non-linear optics.

\section{ACKNOWLEDGMENTS}

We acknowledge financial support from the ONERA through the SONS project.
* patrick.bouchon@onera.fr

1 G. Lerosey, J. de Rosny, A. Tourin, and M. Fink, Science 315, 1120 (2007).

2 A. Grbic, L. Jiang, and R. Merlin, Science 320, 511 (2008).

3 Z. Zhang, R. Peng, Z. Wang, F. Gao, X. Huang, W. Sun, Q. Wang, and M. Wang, Applied Physics Letters 93, 171110 (2008).

${ }^{4}$ L. Novotny and N. van Hulst, Nature Photonics 5, 83 (2011).

5 P. Biagioni, J.-S. Huang, and B. Hecht, Reports on Progress in Physics 75, 024402 (2012).
${ }^{6}$ W. Barnes, A. Dereux, T. Ebbesen, et al., Nature 424, 824 (2003).

7 J.-J. Greffet, Science 308, 1561 (2005)

8 J. Schuller, E. Barnard, W. Cai, Y. Jun, J. White, and M. Brongersma, Nature Materials 9, 193 (2010).

9 T. Siegfried, Y. Ekinci, O. J. F. Martin, and H. Sigg, Nano Letters 13, 5449 (2013), http://pubs.acs.org/doi/pdf/10.1021/nl403030g.

10 M. Knight, H. Sobhani, P. Nordlander, and N. Halas, Science 332, 702 (2011). 


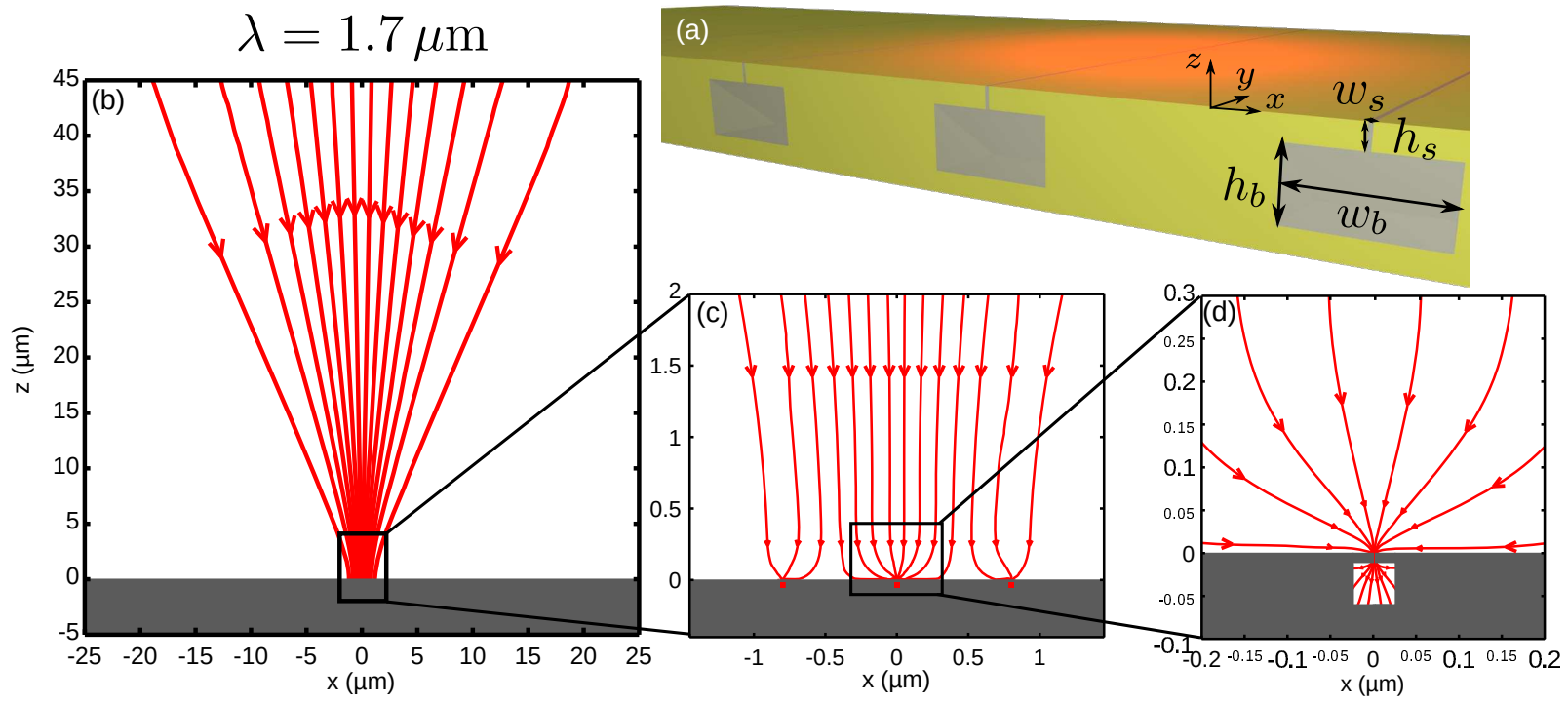

FIG. 6. (a) A beam is focused on the Helmholtz structure with $w_{s}=2.5 \mathrm{~nm}, h_{s}=12 \mathrm{~nm}, w_{b}=h_{b}=49 \mathrm{~nm}$ and $d=800 \mu \mathrm{m}$ (b) Streamlines of the beam focused into the structure, the beam waist is about $\lambda=1.7 \mu \mathrm{m}$ wide. (c) The beam is focused on one individual structure that collects about $60 \%$ of the incoming energy. The remaining energy is fully collected by the neighboring slits. (d) Streamlines of the Poynting vector in the vicinity of the structure, showing that the magneto-electric interference redirects the incident energy inside the resonator ${ }^{24}$.

11 M. Kauranen and A. V. Zayats, Nature Photonics 6, 737 (2012).

12 N. Liu, M. L. Tang, M. Hentschel, H. Giessen, and A. P. Alivisatos, Nature materials 10, 631 (2011).

13 D. Dregely, F. Neubrech, H. Duan, R. Vogelgesang, and H. Giessen, Nature communications 4 (2013).

14 J. Bochterle, F. Neubrech, T. Nagao, and A. Pucci, ACS nano 6, 10917 (2012).

15 R. Adato, A. A. Yanik, J. J. Amsden, D. L. Kaplan, F. G. Omenetto, M. K. Hong, S. Erramilli, and H. Altug, Proceedings of the National Academy of Sciences 106, 19227 (2009).

16 J. A. Schuller, T. Taubner, and M. L. Brongersma, Nature Photonics 3, 658 (2009).

17 P. Mühlschlegel, H. Eisler, O. Martin, B. Hecht, and D. Pohl, Science 308, 1607 (2005).

18 X. Chen, H.-R. Park, M. Pelton, X. Piao, N. C. Lindquist, H. Im, Y. J. Kim, J. S. Ahn, K. J. Ahn, N. Park, et al., Nature communications 4 (2013).

19 L. Novotny, Phys. Rev. Lett. 98, 266802 (2007).

20 T. Kosako, Y. Kadoya, and H. F. Hofmann, Nature Photonics 4, 312 (2010).

${ }^{21}$ D. Dregely, R. Taubert, J. Dorfmüller, R. Vogelgesang, K. Kern, and H. Giessen, Nature communications 2, 267 (2011).

22 P.J. Schuck, D.P. Fromm, A. Sundaramurthy, G.S. Kino, and W.E. Moerner, Physical Review Letters 94, 017402 (2005).
${ }^{23}$ S. Kim, J. Jin, Y.-J. Kim, I.-Y. Park, Y. Kim, and S.-W. Kim, Nature 453, 757 (2008).

${ }^{24}$ F. Pardo, P. Bouchon, R. Haidar, and J.L. Pelouard, Physical Review Letters 107, 093902 (2011).

25 P. Bouchon, F. Pardo, B. Portier, L. Ferlazzo, P. Ghenuche, G. Dagher, C. Dupuis, N. Bardou, R. Haïdar, and J. Pelouard, Applied Physics Letters 98, 191109 (2011).

${ }^{26}$ G. Subramania, S. Foteinopoulou, and I. Brener, Phys. Rev. Lett. 107, 163902 (2011).

27 P. Chevalier, P. Bouchon, R. Haïdar, and F. Pardo, Journal of Nanophotonics 6, 063534 (2012).

${ }^{28} \mathrm{H}$. von Helmholtz, Theorie der Luftschwingungen in Röhren mit offenen Enden, 80 (W. Engelmann, 1896).

29 P. Chevalier, P. Bouchon, R. Haïdar, and F. Pardo, Applied Physics Letters 105, 071110 (2014).

30 E. Palik and G. Ghosh, Handbook of optical constants of solids (Academic press, 1985).

31 P. Bouchon, F. Pardo, R. Haïdar, and J. Pelouard, Journal of the Optical Society of America A 27, 696 (2010).

${ }^{32}$ P. Chevalier, P. Bouchon, F. Pardo, and R. Haidar, Journal of the Optical Society of America A 31 (2014).

33 C. Feuillet-Palma, Y. Todorov, A. Vasanelli, and C. Sirtori, Scientific Reports 3 (2013).

34 M. Seo, H. Park, S. Koo, D. Park, J. Kang, O. Suwal, S. Choi, P. Planken, G. Park, N. Park, et al., Nature Photonics 3, 152 (2009). 